\title{
Las Redes Sociales y su influencia en el Comportamiento de estudiantes de Pregrado de Enfermería
}

\section{Social networks and their influence on the behavior of Nursing Undergraduate students}

Mirtha Sussan Trejo de Ríos, Isidro Javier Ríos Pérez ${ }^{1}$, Jorge Adalberto López Balarezo ${ }^{1}$, Miguel Angel Aguilar Luna Victoria' Carlos Manuel Cruz Castañeda

\section{RESUMEN}

Objetivo: Conocer la influencia de las redes sociales en el comportamiento de los estudiantes de Pregrado de Enfermería de la Universidad Nacional José Faustino Sánchez Carrión Año 2018. Materiales y Métodos: La población estuvo conformada por 280 estudiantes matriculados en el semestre académico 2017-II, se utilizó el muestreo aleatorio simple de 100 estudiantes. Se utilizó la técnica de la encuesta que fueron validadas por el método del Juicio de Expertos y para la confiabilidad se empleó el Coeficiente Alfa de Cronbach. La investigación es aplicada y no experimental. Resultados: El $81 \%$ fueron mujeres y $19 \%$ hombres; sus edades estuvieron comprendidas de 17 y 26 años. El uso de las redes sociales está distribuido: excesivamente 10\%, moderadamente $70 \%$ y escasamente $20 \%$; el comportamiento: bueno $73 \%$, moderado $22 \%$ y bajo $5 \% ; 7 \%$ presentan alta adicción a las redes sociales, $76 \%$ moderada y $17 \%$ baja. Las relaciones interpersonales está distribuida: buena $34 \%$, moderada $64 \%$ y poca $2 \%$; la dependencia a las redes sociales: $13 \%$ bastante, $57 \%$ moderada y $30 \%$ poca y los cambios de personalidad: $7 \%$ bastante, $17 \%$ moderado y $76 \%$ poco. Conclusiones: Existe alta influencia de las redes sociales en el comportamiento, la adicción a las redes sociales en las relaciones interpersonales del comportamiento y la dependencia a las redes sociales en los cambios de personalidad del comportamiento.

Palabras clave: Redes sociales, adicción, cambios de personalidad.

\section{ABSTRACT}

Objective: Know the Influence of social networks on the behavior of undergraduate nursing students of the National University Jose Faustino Sanchez Carrion Year 2018, Materials and Methods: The population consisted of 280 students enrolled in the academic semester 2017-II, simple random sampling of 100 students was used. The survey technique has been used through the instruments the questionnaire that were validated by the Expert Judgment method and for the reliability was used the Alpha Coefficient of Cronbach. The research is applied and not experimental. Results: $81 \%$ were women and $19 \%$ men: their ages were between 17 and 26 years old. The use of social networks is distributed: excessively $10 \%$, moderately $70 \%$ and scarcely $20 \%$; the behavior distributed: good $73 \%$, moderate $22 \%$ and low $5 \% ; 7 \%$ have high addiction to social networks, $76 \%$ moderate and $17 \%$ low. Interpersonal relationships are distributed: good $34 \%$, moderate $64 \%$ and little $2 \%$; dependence on social networks: $13 \%$ quite, $57 \%$ moderate and $30 \%$ little, and personality changes: $7 \%$ quite, $17 \%$ moderate and $76 \%$ little. Conclusions: There is a high influence: from social networks on behavior, addiction to social networks in interpersonal relationships of behavior and dependence on social networks in behavioral personality changes.

Keywords: Social networks, addiction, personality change

\footnotetext{
${ }^{1}$ Facultad de Ciencias. Universidad Nacional José Faustino Sánchez Carrión. Huacho, Perú.

${ }^{2}$ Facultad de Ingeniería Industrial, Sistemas e Informática. Universidad Nacional José Faustino Sánchez Carrión, Huacho, Perú.
} 


\section{INTRODUCCIÓN}

En la actualidad las redes sociales se han convertido, tanto para los jóvenes universitarios como para la sociedad, en uno de los medios de comunicación más utilizados, ya que estas redes son comunidades virtuales conformadas por usuarios, que vienen a ser conocidos de colegios, amigos o compañeros de la universidad, trabajo, amigos en común, etc. Además permite la interacción entre los usuarios de una manera fácil y rápida, asimismo les permite compartir fotos, videos y diferentes recursos haciendo que estas redes crezcan rápidamente, un ejemplo de la misma es el Facebook, sin embargo, el uso inadecuado de las redes sociales presenta desventajas como la adicción, el tiempo que ocupa su uso y su consecuencia en la conducta de los universitarios.

Algunas investigaciones han estudiado el nivel de adicción a las redes sociales que presentan los estudiantes universitarios, Rueda y Flores (2016) encontraron que el porcentaje del nivel de adicción a las redes sociales fue del $23 \%$ sin riesgo, el $18 \%$ riesgo leve, el $29 \%$ riesgo moderado y el $30 \%$ riesgo alto. Asimismo Haussler (2014) aplicó el cuestionario de adicciones a las Nuevas Tecnologías a una población de adolescentes, indicando una prevalencia de $48 \%$ sin adicción, 52\% adicción moderada y 0\% adicción grave. Estos resultados aportan evidencia en cuanto al tema de adicción a las redes sociales en estudiantes universitarios. Molina y Toledo (2014), demuestran que a nivel general, la mayoría de los adolescentes no se ven muy afectados por las redes sociales en las diferentes áreas; aunque dedican hasta tres horas y revisan por lo menos cinco veces al día, además que los distraen de sus estudios.

Por ello el objetivo de la presente investigación fue conocer la influencia de las redes sociales en el comportamiento de los estudiantes de la carrera de Enfermería de la Universidad Nacional José Faustino Sánchez Carrión Año 2018.

\section{MATERIALY MÉTODOS}

La población estuvo conformada por 280 estudiantes de la carrera de Enfermería y se tomó un muestreo aleatorio simple de 100 estudiantes ( $81 \%$ mujeres y $19 \%$ hombres entre 17 y 26 años de edad). El marco de muestreo estuvo constituido por la relación de estudiantes matriculados de la carrera de enfermería. Se aseguró el anonimato de los estudiantes, solicitándole a cada uno de ellos su respectivo consentimiento, para cumplir con la declaración de Helsinky sobre ética de investigación.

Se utilizó un cuestionario conformado por 27 preguntas: el uso de las redes sociales estuvo conformada por las preguntas del 1 al 13 y se les clasificó excesivamente, moderadamente y escasamente y sus dimensiones: adicción las preguntas del 1 al 7 se les clasificó alta, moderada y baja; dependencia conformada por las preguntas del 8 al 13 y se les clasificó bastante, moderada y poca. Por otro lado el comportamiento conformado por las preguntas del 14 al 27 y se les clasificó bueno, moderado y bajo; sus dimensiones; relaciones interpersonales estuvo conformada por las preguntas del 14 al 20 se les clasificó buena, moderada y poca mientras que los cambios de personalidad conformada por las preguntas del 21 al 27 y se les clasificó bastante, moderada y poco (Tabla 1 ).

Se utilizó la estadística descriptiva con el fin de describir fácil y rápidamente las características esenciales de las variables mediante el empleo de métodos gráficos, tabulares o numéricos. Asimismo, se determinó el coeficiente de correlación de Spearman empleando el programa SPSS versión 21. 


\section{RESULTADOS}

Tabla 1. Análisis de la encuesta de las redes sociales y comportamiento de estudiantes de Pregrado de Enfermería

\begin{tabular}{|c|c|c|c|c|c|}
\hline \multirow[b]{2}{*}{ DIM } & \multirow[b]{2}{*}{$\begin{array}{c}\mathbf{N}^{\circ} \\
\text { preg. }\end{array}$} & \multirow[b]{2}{*}{ DESCRIPCIÓN DE LA PREGUNTA } & \multicolumn{3}{|c|}{ RESPUESTAS (\%) } \\
\hline & & & Nunca & $\begin{array}{c}A \\
\text { veces }\end{array}$ & Siempre \\
\hline \multirow{7}{*}{ 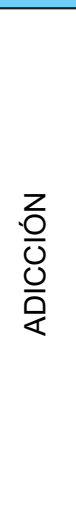 } & 1 & $\begin{array}{l}\text { Me propongo sin éxito, controlar mis hábitos de } \\
\text { uso prolongado e intenso de las redes sociales }\end{array}$ & 21 & 66 & 13 \\
\hline & 2 & $\begin{array}{l}\text { Permanezco mucho tiempo conectado(a) a las } \\
\text { redes sociales }\end{array}$ & 10 & 74 & 16 \\
\hline & 3 & $\begin{array}{l}\text { Aun cuando estoy en clase, me conecto con } \\
\text { disimulo a las redes sociales }\end{array}$ & 12 & 74 & 14 \\
\hline & 4 & $\begin{array}{l}\text { Necesito cada vez más tiempo para atender } \\
\text { mis asuntos relacionados con las redes } \\
\text { sociales }\end{array}$ & 47 & 42 & 11 \\
\hline & 5 & $\begin{array}{l}\text { Cuando entro a las redes sociales pierdo el } \\
\text { sentido de tiempo }\end{array}$ & 51 & 43 & 6 \\
\hline & 6 & $\begin{array}{l}\text { Puedo desconectarme de las redes sociales } \\
\text { por varios días }\end{array}$ & 13 & 63 & 24 \\
\hline & 7 & $\begin{array}{l}\text { Necesito visitar mi red social antes de irme a } \\
\text { dormir }\end{array}$ & 38 & 53 & 9 \\
\hline \multirow{7}{*}{ 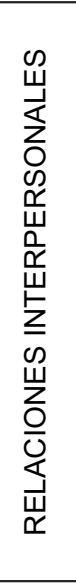 } & 14 & $\begin{array}{l}\text { Mi pareja o amigos o familiares me han llamado } \\
\text { la atención por mi dedicación y el tiempo que } \\
\text { destino a las cosas en las redes sociales }\end{array}$ & 40 & 52 & 8 \\
\hline & 15 & $\begin{array}{l}\text { Aun cuando desarrollo otras actividades, no } \\
\text { dejo de pensar en lo que sucede en las redes } \\
\text { sociales }\end{array}$ & 63 & 32 & 5 \\
\hline & 16 & $\begin{array}{l}\text { Debo controlar mi actividad de conectarme a } \\
\text { las redes sociales }\end{array}$ & 32 & 60 & 8 \\
\hline & 17 & $\begin{array}{l}\text { Estoy atento(a) a las alertas que me envían } \\
\text { desde las redes sociales a mi teléfono o } \\
\text { computadora }\end{array}$ & 13 & 56 & 31 \\
\hline & 18 & $\begin{array}{l}\text { Descuido las tareas y los estudios por estar } \\
\text { conectado(a) a las redes sociales }\end{array}$ & 64 & 33 & 3 \\
\hline & 19 & $\begin{array}{l}\text { Te has distanciado de tus familiares o amigos } \\
\text { debido al uso de las redes sociales }\end{array}$ & 70 & 23 & 7 \\
\hline & 20 & $\begin{array}{l}\text { Temes que la vida sin una red social, sería } \\
\text { aburrida, vacía y sin alegría }\end{array}$ & 57 & 36 & 7 \\
\hline \multirow{7}{*}{ 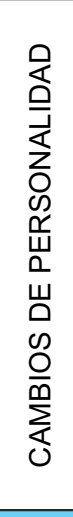 } & 21 & $\begin{array}{l}\text { Me pongo de malhumor si no puedo } \\
\text { conectarme a las redes sociales }\end{array}$ & 78 & 16 & 6 \\
\hline & 22 & $\begin{array}{l}\text { Me siento ansioso(a) cuando no puedo } \\
\text { conectarme a als redes sociales }\end{array}$ & 63 & 30 & 7 \\
\hline & 23 & $\begin{array}{l}\text { Entrar y usar las redes sociales me produce } \\
\text { alivio, me relaja }\end{array}$ & 46 & 41 & 13 \\
\hline & 24 & $\begin{array}{l}\text { Te molestan que te interrumpan cuando estás } \\
\text { conectado(a) }\end{array}$ & 70 & 24 & 6 \\
\hline & 25 & $\begin{array}{l}\text { No sabría qué hacer si se cancelasen todas mis } \\
\text { cuentas online }\end{array}$ & 59 & 27 & 14 \\
\hline & 26 & $\begin{array}{l}\text { Me molesta cuando alguien rechaza mi solicitud } \\
\text { de amistad }\end{array}$ & 83 & 11 & 6 \\
\hline & 27 & $\begin{array}{l}\text { Reaccionas a la defensiva o en secreto cuando } \\
\text { alguien te pregunta que haces en las redes } \\
\text { sociales }\end{array}$ & 69 & 21 & 10 \\
\hline \multirow[b]{2}{*}{ DIM } & $\mathrm{N}^{\mathrm{a}}$ & \multirow[b]{2}{*}{ DESCRIPCIÓN DE LA PREGUNTA } & RES & PUESTA & S (\%) \\
\hline & $\begin{array}{c}\text { PR } \\
\text { G }\end{array}$ & & Nunca & A veces & Siempre \\
\hline \multirow{7}{*}{ 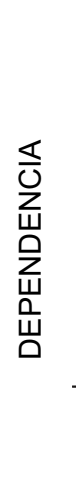 } & 8 & $\begin{array}{l}\text { engo gran necesidad de permanecer } \\
\text { onectado(a) a las redes sociales }\end{array}$ & 21 & 56 & 23 \\
\hline & 9 & $\begin{array}{l}\text { Apenas despierto ya estoy conectándome a las } \\
\text { edes sociales }\end{array}$ & 27 & 53 & 20 \\
\hline & 10 & $\begin{array}{l}\text { a intensidad y la frecuencia con la que entro y } \\
\text { iso la red origina un problema social }\end{array}$ & 38 & 50 & 12 \\
\hline & 12 & $\begin{array}{l}\text { us horas de sueño y tus horas de comida se han } \\
\text { isto alterados por permanecer hablando en tu } \\
\text { ed }\end{array}$ & 53 & 39 & 8 \\
\hline & 13 & $\begin{array}{l}\text { Suando me sucede algo lo público } \\
\text { nmediatamente }\end{array}$ & 73 & 25 & 2 \\
\hline & \multirow[t]{2}{*}{11} & \multirow{2}{*}{$\begin{array}{l}\text { ¿Con qué frecuencia te conectas a las } \\
\text { redes sociales? }\end{array}$} & $\begin{array}{l}<1 \text { hora } \\
\text { diaria }\end{array}$ & $\begin{array}{c}\text { Algunos } \\
\text { días }\end{array}$ & $\begin{array}{l}1 \text { vez por } \\
\text { semana }\end{array}$ \\
\hline & & & 14 & 12 & 72 \\
\hline
\end{tabular}


En la Tabla 1 dimensión adicción, se observó que el $51 \%$ de los estudiantes cuando entran a las redes sociales nunca pierden el sentido de tiempo, el $63 \%$ a veces puede desconectarse por varios días, el $53 \%$ a veces visitan las redes sociales antes de irse a dormir, en la dimensión dependencia se encontró que el $56 \%$ a veces tienen gran necesidad de permanecer conectado(a) a las redes sociales, el $53 \%$ nunca sus horas de sueño y de comida se han visto alterados por permanecer hablando en las redes sociales, en la dimensión relaciones interpersonales indica que el $56 \%$ a veces están atentos(as) a las alertas que les envían desde las redes sociales a su teléfono o computadora, el $64 \%$ nunca descuidan las tareas y los estudios por estar conectado (a) a las redes sociales y el $57 \%$ nunca temen que la vida sin una red social, sería aburrida, vacía y sin alegría y en la dimensión cambios de personalidad se observó que el $63 \%$ nunca se sienten ansioso(a) cuando no pueden conectarse a las redes sociales y el $70 \%$ nunca se molestan que le interrumpan cuando están conectado(a).

Tabla 2. Distribución de los encuestados según el uso de las redes sociales y comportamiento

\begin{tabular}{ccccc}
\hline \multirow{2}{*}{ USO DE LAS } & \multicolumn{3}{c}{ COMPORTAMIENTO } & \multirow{2}{*}{ TOTAL } \\
\cline { 2 - 4 } REDES & Bueno & Moderado & Bajo & \\
\cline { 2 - 4 } SOCIALES & $\%$ & $\%$ & $\%$ & $\%$ \\
\hline Escasamente & 19 & 0 & 1 & 20 \\
Moderadamente & 53 & 16 & 1 & 70 \\
Excesivamente & 1 & 6 & 3 & 10 \\
\hline TOTAL & 73 & 22 & 5 & 100 \\
\hline
\end{tabular}

La Tabla 2 indica que el $20 \%$ de los estudiantes usan escasamente las redes sociales, $70 \%$ moderadamente y $10 \%$ excesivamente mientras que el $73 \%$ presentan buen comportamiento, $22 \%$ moderado y $5 \%$ bajo.

Tabla 3. Distribución de los encuestados según la adicción a las redes sociales y relaciones interpersonales

\begin{tabular}{ccccc}
\hline & \multicolumn{3}{c}{ RELACIONES } & \multirow{2}{*}{ TOTAL } \\
\cline { 2 - 4 } ADICCIÓN & Buena & Moderada & Poca & \\
\cline { 2 - 4 } & $\%$ & $\%$ & $\%$ & $\%$ \\
\cline { 2 - 4 } Baja & 7 & 9 & 1 & 17 \\
Moderada & 26 & 50 & 0 & 76 \\
Alta & 1 & 5 & 1 & 7 \\
\hline TOTAL & 34 & 64 & 2 & 100 \\
\hline
\end{tabular}

En la Tabla 3 se observó que el $17 \%$ de los estudiantes presentan baja adicción a las redes sociales, $76 \%$ moderada y $7 \%$ alta. Del mismo modo el $34 \%$ presenta buena relaciones interpersonales, $64 \%$ moderada y $2 \%$ poca. 
Tabla 4. Distribución de los encuestados según dependencia y cambios de personalidad

\begin{tabular}{ccccc}
\hline & \multicolumn{3}{c}{ CAMBIOS DE PERSONALIDAD } & \multirow{2}{*}{ TOTAL } \\
\cline { 2 - 4 } DEPENDENCIA & Poco & Moderado & Bastante & \\
\cline { 2 - 4 } & $\%$ & $\%$ & $\%$ & $\%$ \\
\hline Poca & 27 & 2 & 1 & 30 \\
Moderada & 45 & 9 & 3 & 57 \\
Bastante & 4 & 6 & 3 & 13 \\
\hline TOTAL & 76 & 17 & 7 & 100 \\
\hline
\end{tabular}

En la Tabla 4 se observó que el $30 \%$ de los estudiantes muestran poca dependencia en las redes sociales, $57 \%$ moderada y $13 \%$ bastante. Por otro lado el $76 \%$ presentan pocos cambios de personalidad, $17 \%$ moderado y $7 \%$ bastante.

Tabla 5. Análisis de las variables usando la prueba de Spearman

\begin{tabular}{lll}
\hline \multicolumn{1}{c}{ Variables } & Valor & Sig. bilateral \\
\hline Redes sociales \& Comportamiento & 0,546 & $4,2 \times 10^{-9}$ \\
\hline Adicción \& Relaciones Interpersonales & 0,449 & 0,000003 \\
\hline $\begin{array}{l}\text { Dependencia \& Cambios de } \\
\text { personalidad }\end{array}$ & 0,492 & $1,97 \times 10^{-7}$ \\
\hline
\end{tabular}

En la Tabla 5 se muestran los $p$-valor (o sig $<0,01$ ) todos menores al nivel de significancia 0,01 ; lo cual indica que existe suficiente evidencia estadística para afirmar que existe alta influencia entre las variables redes sociales y comportamiento, adicción a las redes sociales y relaciones interpersonales así como dependencia a las redes sociales y cambios de personalidad.

\section{DISCUSIÓN}

La mayoría de los estudiantes utilizan frecuentemente el whatsapp resultados diferentes a lo reportado por Molina y Toledo (2014), Rueda y Flores (2016), Salas y Escurra (2014) y Jabalera et al. (2012).

La mayoría de los estudiantes el dispositivo que utilizan para conectarse a internet es el celular; están conectados más de una hora diaria a las redes sociales, lo que motiva a registrarse en las redes sociales es hacer amigos y comunicarse con familia en el extranjero, no publican inmediatamente cuando les sucede algo, nunca se han distanciado de sus familiares o amigos debido al uso de las redes sociales y no tienen problemas familiares estos resultados concuerdan con Molina y Toledo (2014).
La mayoría de los estudiantes a veces tienen gran necesidad de permanecer conectado(a) a las redes sociales, a veces cuando están en clases se conectan a las redes sociales y nunca se muestran ansiosos y malhumorados cuando no pueden conectarse a las redes sociales resultados diferentes hallados por Molina y Toledo (2014), siempre aceptan cualquier tipo de solicitud de amistad resultados similares a lo hallado por Bolaños (2015) y diferentes a lo reportado por Molina y Toledo(2014).

Los estudiantes mayoritariamente muestran un nivel de adicción moderado resultados diferentes a lo reportado por Rueda y Flores (2016) y Castellano (2017). El uso de las redes sociales influye en el comportamiento de los estudiantes resultado que no concuerda con lo reportado por Molina y Toledo (2014) mientras son similares a lo realizado por 
Jabalera et al. (2012). La adicción a las redes sociales influye en las relaciones interpersonales del comportamiento de los estudiantes de la carrera de Enfermería. La dependencia a las redes sociales influye en los cambios de personalidad del comportamiento de los estudiantes de la carrera de Enfermería.

Hoy, en día las redes sociales se han convertido en uno de los principales medios de comunicación e información entre los universitarios de nuestra sociedad, de esta forma se mantienen informados de lo que ocurre a su alrededor y entretenidos, ya que se han convertido en una gran fuente de ocio y de poder. Generalmente, se consideran como "servicios basados en la web que permiten a sus usuarios relacionarse compartir información, coordinar acciones y en general mantenerse en contacto" (Orihuela, 2008).

Esta comunicación virtual se realiza con el fin de comunicarse de una forma instantánea y rápida entre personas distanciadas prefiriendo la pantalla y el teclado haciendo que las relaciones interpersonales se vean deterioradas, ya que dichas relaciones consisten en la comunicación y contacto físico entre personas con el fin de obtener información en relación con el entorno al que pertenecen. Según Cachia (2008) es más fácil contactar con diferentes personas sin ser necesario establecer una relación muy afianzada se crean unas relaciones más débiles, en las que no existe una conexión emocional pero si un intercambio de información, es decir se crean unas conexiones más sueltas y menos comprometidas en las que no hace falta mucho tiempo y esfuerzo.

\section{REFERENCIAS BIBLIOGRÁFICAS}

Bolaños, M. (2015). Estudio del Impacto de las Redes Sociales en el Comportamiento de los Adolescentes de 12 a 14 años de una Unidad Educativa en la Ciudad Guayaquil. Tesis para la obtención del Título de Ingeniero de Sistemas. Universidad Salesiana Sede Guayaquil.

Cachia, R. (2008). Los sitios de creación de redes. Telos: Cuadernos de comunicación e innovación, 76: 69-74.

Castellano, L. (2017). Relaciones Interpersonales y Adicción a Redes Sociales en Estudiantes de una Universidad Privada de Lima Metropolitana. Tesis para optar el Título Profesional de Licenciada en Psicología. Universidad Ricardo Palma.

Jabalera, P., Morey, M., Rodríguez, A. y Sánchez, A. (2012). Las Redes Sociales influyen en el Comportamiento de los Universitarios. Revista electrónica de Investigación Docencia Creativa 1: 88-94. España.

Orihuela, J. (2008). Internet: la hora de las redes sociales. Nueva Revista de Política, Cultura y Arte, 119: 57-62.

Molina, G. y Toledo, R. (2014). Las Redes Sociales y su Influencia en el Comportamiento de los Adolescentes. Tesis para la obtención del título Licenciado en Psicología Clínica. Universidad del Azuay CuencaEcuador.

Rueda y Flores (2016). Adicción a las redes sociales en estudiantes universitarios. Revista Tlamati Sabiduría 7(1): 512525. México.

Correo electrónico: mstl@hotmail.com

Revisión de pares: 15-04-2019

Recibido: 22-06-2019 